\title{
因HAD
}

DOI: http://doi.org/10.22585/hospdomic.v3i4.88

\section{Análisis bibliométrico de los ensayos clínicos, indizados hasta el año 2018, en la base bibliográfica Cochrane Library realizados en el ámbito de la hospitalización a domicilio}

Bibliometric analysis of clinical trials, indexed up to 2018 , in the Cochrane Library bibliographic database performed in the field of hospital-based home care services

Vanessa Esperanza Oller-Arlandis', Javier Sanz Valero',2, Carmina Wanden-Berghe ${ }^{3}$

1. Universidad Miguel Hernández de Elche. Departamento de Salud Pública e Historia de la Ciencia. Alicante, España.

2. Instituto de Salud Carlos III. Escuela Nacional de Medicina del Trabajo. Madrid, España.

3. Hospital General Universitario de Alicante, Instituto de Investigación Sanitaria y Biomédica de Alicante (ISABIAL-FISABIO). Alicante, España.

Correspondencia/Correspondence

Vanessa Esperanza Oller-Arlandis

vaneollerarlandis@gmail.com

Recibido/Received

24.08.2019

Aceptado/Accepted

30.09.2019
Conflicto de Intereses/Competing interest Las autoras y el autor del trabajo declaran no tener ningún conflicto de interés

CÓMO CITAR ESTE TRABAJO | HOW TO CITE THIS PAPER

Oller-Arlandis VE, Sanz Valero J, Wanden-Berghe C. Análisis bibliométrico de los ensayos clínicos, indizados hasta el año 2018, en la base bibliográfica Cochrane Library realizados en el ámbito de la hospitalización a domicilio. Hosp Domic. 2019;3(4):255-68 


\section{RESUMEN}

Objetivo: Analizar y caracterizar, mediante técnica bibliométrica, los ensayos clínicos realizados en el ámbito de la hospitalización a domicilio, indizados en la base de datos bibliográfica Cochrane Library.

Método: Análisis bibliométrico. Los datos se obtuvieron de la base de datos Cochrane, empleando el MeSH, «Home Care Services, Hospital-Based». Fecha de la búsqueda agosto de 2019.

Resultados: Se analizaron 224 referencias. El número de ensayos clínicos controlados aleatorizados fue 213 referencias $(95,1 \%)$, con un total de 253 pertenecientes a 161 instituciones. El idioma predominante fue el inglés con 215 casos (96,0\%). La obsolescencia presentó mediana de 11 años (Índice de Burton-Kebler) e Índice de Price del 19,2\%. Se constató que 223 (99,6\%) ensayos estaban registrados mediante el identificador documental de PubMed. Según el Medical Subject Headings (MeSH) principal presentó un total de 128 temas diferentes, siendo la Enfermedad Pulmonar Obstructiva Crónica $(n=24,10,7 \%)$ la más presente.

Conclusiones: Los indicadores métricos obtenidos están en línea con otras áreas de conocimiento relacionadas con las ciencias de la salud. La publicación de ensayos clínicos sobre $\mathrm{HaD}$ se mantiene constante durante el tiempo, sin identificarse grandes grupos de referencia. Los descriptores y las áreas temáticas son acordes al área estudiada y los ensayos están publicados en revistas relacionadas con el tema a estudio, aunque sería conveniente, tener una mayor presencia en publicaciones referentes sobre HaD.

Palabras clave: Ensayo Clínico; Servicios de atención a domicilio provisto por hospital; Bibliometría; Acceso a la Información; Indicadores bibliométricos; Cochrane Library.

\section{ABSTRACT}

Objective: Analyze and characterize, through bibliometric technique, clinical trials conducted in the field of home hospitalization, indexed in the bibliographic database the Cochrane Library.

Method: Bibliometric analysis. Data were obtained from the Cochrane database, using the $\mathrm{MeSH}$, "Home Care Services, Hospital-Based". The search date was August 2019.

Results: 224 references were analyzed. The number of randomized controlled clinical trials was 213 references (95.1\%), with a total of 253 belonging to 161 institutions. The predominant language was English with 215 cases (96.0\%). The obsolescence presented a median of 11 years (Burton-Kebler Index) and the Price Index was $19.2 \%$. It was found that 223 (99.6\%) clinical trials were registered using the PubMed documentary identifier. According to the main Medical Subject Headings (MeSH) it presented a total of 128 different subjects, with Chronic Obstructive Pulmonary Disease ( $n=24,10.7 \%$ ) being the most present.

Conclusions: The metric indicators obtained are in line with other areas of knowledge related to health sciences. The publication of clinical trials on $\mathrm{HaH}$ remains constant over time, without identifying large reference groups. The descriptors and thematic areas are consistent with the area studied and the essays are published in journals related to the subject under study, although it would be convenient to have a greater presence in reference publications on $\mathrm{HaH}$.

Keywords: Clinical Trial; Home Care Services, Hospital Based; Bibliometrics; Access to information; Bibliometric indicators; Cochrane Library. 


\section{INTRODUCCIÓN}

El crecimiento de la producción científica en las últimas décadas y su indización en bases de datos bibliográficas automatizadas han potenciado el uso de la Bibliometría y la generación de indicadores para medir los resultados de la actividad científica y tecnológica. El estudio empírico de la actividad científico-investigativa a partir de los artículos científicos publicados se basa en la aplicación de métodos cuantitativos, basados en indicadores y modelos matemáticos, que permiten caracterizar su desarrollo y evolución (1,2). Mediante los indicadores bibliométricos se pueden evaluar las revistas y otras publicaciones periódicas de manera objetiva, observar las tendencias que siguen y generar información útil para mejorar su gestión (3).

Así pues, estos estudios son una herramienta esencial para describir y evaluar los resultados de las investigaciones que se divulgan a través de las revistas científicas (4). La utilización de indicadores bibliométricos, para evaluar la actividad científica, descansa en la premisa de que las publicaciones son el resultado de la investigación y que, por lo tanto, de la observación, análisis y evaluación de sus publicaciones podemos inferir la situación de la actividad científica (5).

Por otro lado, la evidencia científica es una medida que nos sirve para determinar cuánto de fiables son los datos, resultados y, por tanto, las conclusiones de las investigaciones clínicas. Si una investigación clínica es de baja evidencia científica puede ocurrir que los datos generados en ella, no sean fiables y nos induzcan a conclusiones erróneas, lo que puede provocar, que una vez obtenida una conclusión, pueda ser refutada por no ser veraz (6).

El grado de evidencia científica de los estudios de investigación se valora mediante escalas que permiten clasificarlos de manera jerárquica según el rigor del diseño. Las recomendaciones para la práctica realizadas a partir de estos estudios, dependen de su metodología, del rigor científico. Este, a su vez, se asocia a la asignación aleatoria de los grupos control y experimental, a la existencia de un grupo control concurrente en el tiempo, al sentido prospectivo del estudio, al enmascaramiento de participantes e investigadores y al tamaño de muestra, suficientemente grande para detectar diferencias significativas (7). En esta sentido, el ensayo clínico representa un nivel de evidencia lb y un grado de recomendación B según la U. S. Agency for Healthcare Research and Quality (8).

Por ello, el estudio de la producción científica en un área temática determinada, en este caso los ensayos clínicos sobre el hospital a domicilio $(\mathrm{HaD})$, es un buen indicador de la progresión de la investigación y de la generación de conocimientos sobre HaD. Su análisis bibliométrico permite un examen retrospectivo sobre el modo que ha sido investigada y dada a conocer, pero también puede evaluar el potencial de investigación de los grupos e instituciones involucradas (9).

En consecuencia, el objetivo de este trabajo fue analizar y caracterizar, mediante técnica bibliométrica, los ensayos clínicos realizados en el ámbito de la hospitalización a domicilio, indizados en la base de datos bibliográfica Cochrane Library.

\section{MATERIAL Y MÉTODO}

\section{Diseño}

Estudio descriptivo trasversal de la producción científica de los ensayos clínicos (EC) sobre hospitalización a domicilio indizados en la base bibliográfica Cochrane Library. 


\section{Fuente de obtención de los datos}

Se obtuvieron de la consulta directa y acceso, vía Internet, a la documentación científica existente en la base de datos.

\section{Unidad de análisis}

Los ensayos clínicos sobre hospitalización a domicilio indizados, en la base de datos bibliográfica indicada, hasta el año 2018.

\section{Búsqueda bibliográfica}

A partir de la consulta al Medical Subject Headings (MeSH), Thesaurus desarrollado por la U.S. National Library of Medicine, se utilizó el descriptor "Home Care Services, Hospital-Based"[MeSH]. Realizando la búsqueda en la pestaña "Medical Terms (Mesh)" desde el apartado Advanced Search del buscador adscrito a la base bibliográfica [https://www.cochranelibrary.com/advancedsearch/mesh].

La búsqueda se realizó en agosto de 2019.

\section{Indicadores a estudio}

Producción científica: calculada según número de artículos indizados.

Tipología documental: clasificación según el tipo de EC.

Población incluida en los EC: clasificada en 5 grupos: Adultos en general (19 y más años); Ancianos (más de 65 años); Adultos (de 19 a 95 años); Niños y adolescentes (de 1 a 18 años); Neonatos (nacidos de menos de 1 año).

Edad de los EC: 2018 menos año de publicación del artículo.

Semiperiodo de Burton-Kebler (o Índice de Burton-Kebler): mediana de la distribución del conjunto de las referencias ordenadas por su antigüedad).

Índice de Price: porcentaje de referencias, sobre EC, con edad menor de 5 años.

Idioma de publicación del EC.

Distribución geográfica de procedencia de los artículos.

Índice de colaboración institucional: número de instituciones que figuran como firmantes del documento.

Filiación institucional del primer firmante e Índice de Lotka: las instituciones se agruparon en tres niveles de rendimiento: pequeños productores (Índice de transitoriedad = un único trabajo), medianos productores (entre 2 y 9 trabajos) y grandes productores (10 o más trabajos).

Número de autores por artículo e índice de colaboración (cociente entre el número de firmas y el número de trabajos).

Revista donde se publica el artículo.

Impacto de las publicaciones según el Journal Citation Report Science Edition Database (JCR) y del Scimago Journal Rank (SJR).

Dispersión: núcleo principal de Bradford (conjunto de revistas de mayor pertinencia para un área del conocimiento).

Campos de identificación documental: 
1. PM (campo de registro en la base de datos Cochrane correspondiente al PMID -PubMed Identifier-): identificador único asignado a cada referencia de un artículo recogido en la base de datos bibliográfica MEDLINE.

2. XR (campo de registro en la Cochrane correspondiente al Embase Identification Number): identificador único de cada referencia de un artículo en la base de datos bibliográfica Embase.

3. DOI: digital object identifier; sistema de identificación (similar a los identificadores URI) que permite localizar, a través de la Red, el documento caracterizado mediante este código.

Temática de los EC: Descriptor (MeSH), como Major Topic, que representan el contenido principal de cada EC.

\section{Análisis de los datos}

Las variables cualitativas se describieron por su frecuencia y relativa (porcentaje), las cuantitativas mediante su Media y Desviación Estándar, con Intervalos de Confianza del 95\%; representando las más relevantes mediante la utilización de tablas y gráficos. Se utilizó la mediana y la moda, como medidas de tendencia central, calculándose en ocasiones, el máximo y el mínimo. Para conocer la evolución de algunos indicadores se segmentó el período de estudio en dos etapas, tomando como punto de corte el Índice de Burton-Kebler (la mediana): 1. ${ }^{a}$ época: de 1994 a 2007 y $2 .{ }^{a}$ época: de 2008 a 2018. El crecimiento de la producción científica se examinó mediante el análisis de regresión. La existencia de asociación entre variables cualitativas se analizó mediante la prueba de chi-cuadrado de Pearson. Para comprobar la significación en la diferencia de medias para muestras independientes se utilizó la prueba t de Student. El nivel de significación utilizado en todos los contrastes de hipótesis fue $a \leq 0,05$.

Para el almacenamiento de datos y análisis estadístico se empleó el programa Statistical Package for the Social Sciences (IBM-SPSS), versión 22 para Windows.

El control de la calidad de la información se efectuó mediante la doble entrada de los datos, corrigiendo las inconsistencias mediante la consulta con los datos originales.

\section{RESULTADOS}

\section{Producción científica}

La búsqueda realizada dio un total de 224 referencias. El primer EC indizado corresponde al año 1994, publicado en la revista Obstetrics and Gynecology (10) y que fue incluido en Cochrane Central Register of Controlled Trials el 31 de enero de 1998. El año 2008 fue el de mayor producción con $15(6,7 \%)$ EC.

Al estudiar la relación entre el número de publicaciones por año se comprobó que el modelo de regresión con un mayor ajuste fue el exponencial creciente, siendo el coeficiente de determinación $R 2=0,1(p<0,001)$; ver figura 1 . 
Figura 1. Evolución anual de la producción científica de los ensayos clínicos Hospital a Domicilio, recuperada de la base de datos bibliográfica The Cochrane Library y su ajuste a la regresión lineal

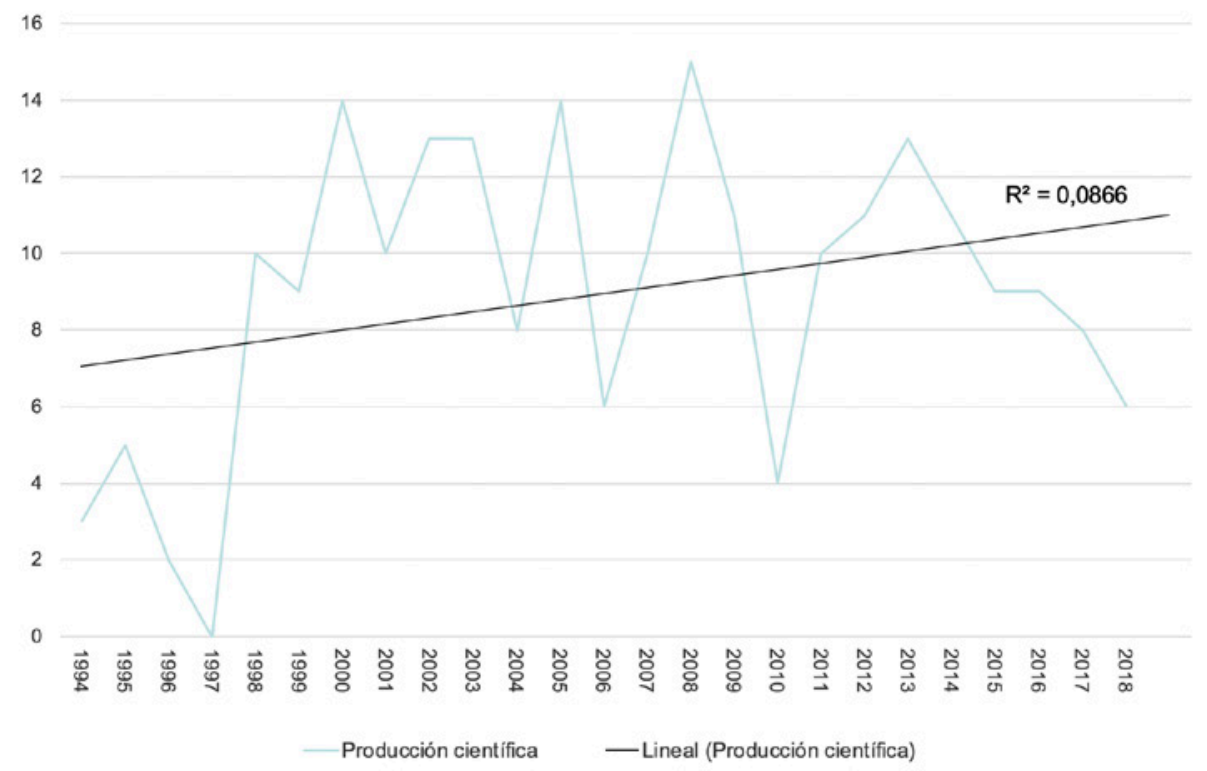

\section{Obsolescencia/actualidad de la producción científica}

La edad media de los EC analizados dio un valor de 11,1 $\pm 0,4$ años, con mediana de 11 años (Índice de Burton-Kebler) y máximo de 24 años. El Índice de Price obtenido fue del 19,2\%.

\section{Tipología documental y población incluida}

La mayoría de los EC presentaron un diseño de ensayo clínico controlado aleatorizado, 213 referencias $(95,1 \%)$, siendo el otro tipo observado el de ensayo clínico controlado con $11(4,9 \%)$ referencias.

No existió asociación significativa, relacionadas con la tipología documental, entre las dos épocas a estudio (chi cuadrado de Pearson $=1,9 ; \mathrm{gl}=1 ; \mathrm{p}=0,163$ ).

La población incluida en los EC presentó la distribución que se recoge en la tabla 1, no observándose asociación, según época a estudio, en relación a la población incluida en los EC (chi cuadrado de Pearson $=3,4 ; g l=4 ; p=0,488$ ). 
Tabla 1. Distribución de la población estudiada en los ensayos clínicos indizados en la base Cochrane Library sobre Hospital a Domicilio

\begin{tabular}{|l|l|l|}
\hline & Núm. & porcentaje \\
\hline Adultos en general (19 y más años) & 99 & 44,2 \\
\hline Ancianos (más de 65 años) & 56 & 25,0 \\
\hline Adultos (de 19 a 95 años) & 47 & 21,0 \\
\hline Niños y adolescentes (de 1 a 18 años) & 18 & 8,0 \\
\hline Neonatos (nacidos de menos de 1 año) & 4 & 1,8 \\
\hline
\end{tabular}

\section{Autoría}

Se contabilizaron un total de 253 autores, con un máximo de 93 autores observado en un EC. La media, equivalente al Índice de Cooperación, fue de 6,7 $\pm 0,4$ autores/referencia. La mediana fue de 6 autores por artículo y la moda de 1 autor/referencia.

La evolución del número de autores por artículo fue ascendente a lo largo de los años, ajustándose a un modelo de curva exponencial con un coeficiente de determinación $R 2=0,2$ y $p<0,001$. Así, en la 2. ${ }^{a}$ época a estudio, el cociente autores/referencia fue superior al observado en la $1 .{ }^{2}$ época: 8,3 versus $5,2, p=0,001$.

\section{Procedencia geográfica, filiación institucional e idioma}

El país del autor que figuraba como primer firmante, fue preferentemente el Reino Unido con $42(18,8 \%)$ referencias. Otros países que publicaron más de 10 EC fueron: Estados Unidos con 32 (14,3\%), Australia con 29 (12,9\%), Italia y Canadá con 15 (6,7\%) y Suecia y Francia con 12 (5,4\%). Los documentos con primera filiación española fueron $9(4,0 \%)$. En $4(1,8 \%)$ documentos no se consignó el país de filiación.

Los países con más de 3 EC, ordenados según época, pueden consultarse en la tabla 2, obteniéndose diferencias significativas entre las dos épocas a estudio (chi cuadrado de Pearson $=41,7$; $g l=27 ; p=0,035$. 
Tabla 2. Distribución por países de los ensayos clínicos indizados en la base Cochrane Library sobre Hospital a Domicilio, según época de estudioa

\begin{tabular}{|c|c|c|c|c|}
\hline \multirow[t]{2}{*}{ País } & \multicolumn{2}{|c|}{$\begin{array}{l}1^{\text {a }} \text { época } \\
1994 \text { a } 2007\end{array}$} & \multicolumn{2}{|c|}{$\begin{array}{l}\text { 2. }{ }^{a} \text { época } \\
2008 \text { a } 2018\end{array}$} \\
\hline & núm. & $\%$ & núm. & $\%$ \\
\hline Reino Unido & 27 & 12,1 & 15 & 6,7 \\
\hline Estados Unidos & 22 & 9,8 & 10 & 4,7 \\
\hline Australia & 15 & 6,7 & 14 & 6,3 \\
\hline Italia & 5 & 2,2 & 10 & 4,7 \\
\hline Canadá & 8 & 3,6 & 7 & 3,1 \\
\hline Suecia & 6 & 2,7 & 6 & 2,7 \\
\hline Francia & 7 & 3,1 & 5 & 2,2 \\
\hline España & 3 & 1,3 & 6 & 2,7 \\
\hline
\end{tabular}

Se observó colaboración internacional, entre 2 o más países, en 10 (4,5\%) EC; hallándose diferencias en las medias del número de países/documento entre las dos épocas estudiadas (1,0 versus 1,2; t de Student $=-2,6 ; \mathrm{gl}=106 ; \mathrm{p}=0,011$ ).

Se identificaron trabajos publicados de 161 instituciones, con una mediana de 1 centro por referencia. La media del número de instituciones por artículo -índice de colaboración institucional- fue de 1,5 \pm 0,1; encontrando diferencias en las medias del índice de colaboración institucional entre las dos épocas estudiadas $(1,0$ versus $2 ; t$ de Student $=-4,0 ; g l=106 ; p<0,001)$. Un total de 36 $(16,1 \%)$ EC fueron realizados en colaboración entre 2 o más centros.

La filiación de los documentos analizados se clasificó, extrapolando el Índice de Lotka, en tres niveles de rendimiento: pequeños productores, o Índice de Transitoriedad (un único trabajo), donde encontramos 123 centros (76,4\%); medianos productores (entre 2 y 9 trabajos) con 33 centros $(20,5 \%) ; y$ grandes productores (10 o más trabajos) donde no se localizó ninguna institución. En 5 $(3,1)$ referencias no figuraba filiación.

El idioma predominante de las referencias recuperadas fue el inglés, con 215 casos (96,0\%). Dejar constancia del resto de idiomas utilizados en los EC que fueron, francés con $3(1,3 \%)$ trabajos, español con $2(0,9 \%)$ y sueco, japonés, coreano y alemán con $1(0,4 \%)$ EC. No se encontraron diferencias significativas entre las dos épocas a estudio (chi cuadrado de Pearson $=8,6 ; \mathrm{gl}=6 ; \mathrm{p}$ $=0,197)$. 


\section{Revistas, dispersión e impacto}

Se recuperaron un total de 127 revistas. El estudio de la dispersión de la literatura científica recuperada, determina la concentración de una frecuencia similar de documentos en un número diferente de revistas: núcleo principal, con 12 revistas (9,4\%) que incluían 73 EC (32,6\%). La zona 2 con 40 revistas (31,5\%) que contenían 75 EC (33,9\%) y la zona 3 con 75 revistas $(59,1 \%)$ que englobaban 76 EC (33,8\%); ver figura 2: gráfico de dispersión de Bradford.

Figura 2: Dispersión de las revistas y los ensayos clínicos (EC) sobre Hospital a Domicilio en la base de datos bibliográfica Cochrane Library

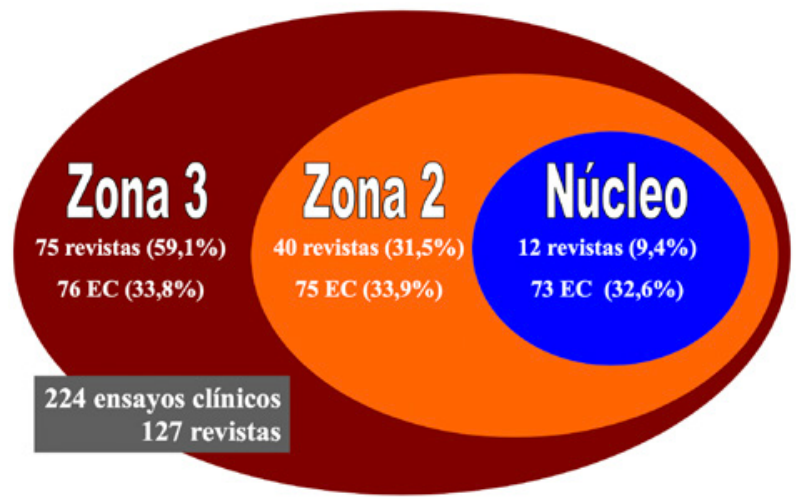

Como puede observarse en la tabla 3, las revistas que publicaron 5 o más artículos sobre el tema a estudio fueron 6 , todas ellas presentes en las principales bases de datos sobre impacto de las publicaciones.

Tabla 3. Revistas indizadas en Cochrane Library que han publicado 5 o más ensayos clínicos sobre Hospital a Domicilio

\begin{tabular}{|c|c|c|c|c|c|c|}
\hline Abreviatura de la revista & $f_{0}$ & $\%$ & $\mathrm{FI}_{(\mathrm{JCR})}$ & $\mathbf{Q}_{\text {(JCR) }}$ & SJR & $\mathbf{Q}_{(\mathrm{SJR})}$ \\
\hline Clinical Rehabilitation & 11 & 8,7 & 2,738 & Q1 & 3,697 & Q1 \\
\hline Journal of the American Geriatrics Society & 11 & 8,7 & 4,113 & Q1 & 2,132 & Q1 \\
\hline British Medical Journal (BMJ) & 10 & 7,9 & 2,376 & Q2 & 2,881 & Q1 \\
\hline Trials & 9 & 7,1 & 1,975 & Q3 & 1,287 & Q1 \\
\hline Respiratory medicine & 6 & 4,7 & 3,237 & Q2 & 1,456 & Q1 \\
\hline Thorax & 5 & 3,9 & 9,640 & Q1 & 1,338 & Q1 \\
\hline \multicolumn{7}{|c|}{$\begin{array}{l}\mathrm{f}_{0}=\text { Número de artículos publicados; FI(JCR) = Factor de Impacto según la base de datos Journal Citation Report para } 2018 \\
Q(J C R)=\text { Cuartil según la mejor posición que ocupa la revista en cualquier categoría en la base de datos Journal Citation Report } \\
\text { SJR = Indicador Scimago Journal Rank para 2018; Q(SJR) = Cuartil según la mejor posición que ocupa la revista en cualquie } \\
\text { categoría en la base de datos Scimago Journal \& Country Rank. }\end{array}$} \\
\hline
\end{tabular}




\section{Campos de identificación documental}

Se constató que 223 (99.6\%) EC estaban registrados mediante PM, 60 (26,8\%) identificaban el campo de registro XR y $183(81,7 \%)$ disponían de DOI. No se probó asociación significativa entre las dos épocas a estudio en el caso del campo de registro PM (chi cuadrado de Pearson $=1,1 ; \mathrm{gl}$ $=1 ; p=0,295$ ) e igual para el campo XR (chi cuadrado de Pearson $=1,1 ; g l=1 ; p=0,918$ ). Por el contrario, si hubo diferencias significativas en relación a la presencia del DOI a favor de la 2. época $(45,5 \%$ versus $36,2 \%$; chi cuadrado de Pearson $=24,5 ; \mathrm{gl}=1 ; \mathrm{p}<0,001)$.

\section{Temática de los ensayos clínicos}

Para conocer la temática de la producción científica a estudio se comprobó que las 224 referencias analizadas habían sido indizados mediante 128 diferentes Medical Subject Headings (MeSH) como Major Topic. La temática más estudiada y presente en 10 o más EC puede consultarse en la tabla 4. Al mismo tiempo, 23 (10,3\%) EC utilizaron la telemedicina para el desarrollo del EC.

Tabla 4. Temática según los Medical Subject Headings, como Major Topics, utilizados 5 o más veces en la indización de los en los ensayos clínicos indizados en la base Cochrane Library sobre Hospital a Domicilio

\begin{tabular}{|l|l|l|}
\hline & Núm. & porcentaje \\
\hline Enfermedad Pulmonar Obstructiva Crónica & 24 & 10,7 \\
\hline Insuficiencia Cardíaca & 21 & 9,3 \\
\hline Rehabilitación de Accidente Cerebrovascular & 13 & 5,8 \\
\hline Artroplastia de Reemplazo de Rodilla & 8 & 3,6 \\
\hline Enfermedad Coronaria & 7 & 3,1 \\
\hline Enfermedades Pulmonares Obstructivas & 6 & 2,7 \\
\hline Costos de la Atención en Salud & 6 & 2,7 \\
\hline Fracturas de Cadera & 5 & 2,2 \\
\hline Puente de Arteria Coronaria & 5 & 2,2 \\
\hline Cuidado Terminal & 5 & 2,2 \\
\hline
\end{tabular}

No existieron diferencias, en cuanto a la temática principal de los EC entre las dos épocas a estudio (chi cuadrado de Pearson $=123,4 ; \mathrm{gl}=102 ; \mathrm{p}=0,074$ ). Si se observaron diferencias sig- 
nificativas en relación a la aplicación de la telemedicina a favor de la 2. a época (8,1\% versus 2,2\%; chi cuadrado de Pearson = 9,6; $g l=1 ; p=0,002$ ).

\section{DISCUSIÓN}

El presente trabajo analiza los principales indicadores bibliométricos de la producción científica sobre los ensayos clínicos indizados en la base de datos Cochrane sobre hospitalización a domicilio. Según el análisis efectuado, los documentos recuperados están en consonancia con la temática seleccionada.

En esta producción se observó un progresivo incremento de los documentos publicados, que si bien logró un modelo de crecimiento exponencial significativo, con acusados vaivenes, este no presentó incrementos importantes. Quizá, este hecho se deba al seleccionar un diseño altamente específico (ensayos clínicos) en un área temática (la HaD) que per se no ha alcanzado la "explosión de la información" $(2,11)$.

En relación a la tipología documental observada, no es de extrañar que prácticamente la totalidad de los ensayos recuperados, en las dos épocas a estudio, fueran controlados aleatorizados, debido a las características de indización que impone la base de datos bibliográfica Cochrane y propio de un área inminentemente clínica (12).

Que la población estudiada en los ensayos clínicos fuera primordialmente adulta fue esperado, ya que solamente el 30\% de los medicamentos comercializados en Europa incluyen autorización pediátrica y menos del $50 \%$ de los fármacos autorizados en niños han sido adecuadamente testados en la población infantil. Esto es debido, en gran medida, a la elevada complejidad y a los requisitos de excelencia que son necesarios para hacer dichos estudios (13).

El número de autores/ensayo clínico presentó datos similares al de otros estudios bibliométricos sobre $\mathrm{HaD}$ (2). Ahora bien, la evolución en el tiempo del número de autores por documento es un dato alentador, aunque mejorable, en cuanto a las autorías colectivas y a la formación de grupos de trabajo (14).

La procedencia mayoritaria de artículos con filiación anglófona es un hecho conocido y ya recogido cumplidamente en la literatura científica $(2,11,15,16)$. Igualmente, se conoce que el predominio del inglés es una constante en las publicaciones de las ciencias de la salud. El idioma de publicación es importante por cuanto se ha comprobado que, cualquiera sea la naturaleza de un artículo científico, las posibilidades de recibir citaciones son mayores si se publica en inglés (17).

No se localizaron artículos de grandes grupos de investigación -con más de 10 publicaciones sobre la materia- lo que puede lastrar la visibilidad y el impacto de la materia estudiada (18). Es positivo, que en la segunda época a estudio existiera una mayor colaboración institucional con respecto a la primera, pero con valores por debajo a lo esperado $(2,19)$. En esta línea, sería interesante conocer el presupuesto que se invierte en Investigación y Desarrollo (I+D) en la temática a estudio. Quizá este dato nos diera un índice ajustado de la producción de cada país o institución en esta categoría del conocimiento (1).

Las revistas que contienen un mayor número de ensayos clínicos sobre HaD no presentan un enfoque exclusivo en la temática, lo que puede explicar a su vez la amplia dispersión que se ha observado en este estudio. La presencia de publicaciones en revistas de alto impacto, indizadas en la Journal Citation Report, era esperable y lógico con el fin de conseguir la mayor visibilidad (y citación). Aunque, cuando se comprobó la temática publicada (propia de la hospitalización domiciliaria), esta concordó con los títulos de las revistas donde se publicaron los ensayos. 
Es significativo la presencia de códigos de identificación documental, fundamentalmente del PM (PMID: PubMed Identifier) ya que este identificador permite un acceso rápido y persistente al documento primario en la base de datos bibliográfica MEDLINE (a través de PubMed). Por ende, que prácticamente la totalidad de ensayos analizados tuvieran este código facilitó la recuperación de los documentos sobre HaD.

La utilización del Digital Object Identifier (DOI), similar a un identificador de recursos uniforme (URI), señaló su indiscutible implantación y uso (20). Además, que hubiera una mayor presencia del DOI en los ensayos, en la segunda época estudiada, es un hecho que contribuye y mejora el acceso a la fuente primaria. La baja presencia del XR (identificador de la base Embase) es extraño y se pudo deber a la poca vinculación que existe entre esta base y la Cochrane, situación que sí que ocurre entre esta última y MEDLINE.

La utilización del Descriptor MeSH (en este caso, como Major Topic) sirvió para clasificar los trabajos indizados y facilitar la categorización temática de los ensayos que no hubiera sido posible tan solo a través del título o del resumen del trabajo (16). Los resultados sobre la clasificación temática de la producción científica muestran unos datos altamente relacionados con el área de conocimiento analizada, representada fundamentalmente por el descriptor «Home Care Services, HospitalBased» e indican un excelente porcentaje, en cuanto a la indización, en las dos épocas analizadas. La introducción de este Descriptor, en el tesauro de la U.S. National Library of Medicine, permite realizar adecuadamente la recuperación de esta documentación y demuestra la adecuación de la ecuación de búsqueda empleada para la consecución de esta información de manera precisa.

Posibles limitaciones de este trabajo. Si bien, se ha utilizado el autor designado para la correspondencia para estudiar la filiación institucional y esto puede ir en detrimento del resto de los autores del artículo, y ser un posible sesgo, existen trabajos que han analizado la no existencia de diferencias significativas entre tomar solo este autor o la totalidad de ellos (2,21). No se estudió la relación entre el número de autores por artículo y el número de citas ya que trabajos previos refieren una débil relación (22) o, incluso, la no existencia de diferencias significativas (23).

Por todo lo anteriormente expuesto, se puede concluir: Ios indicadores métricos obtenidos están en línea con otras áreas de conocimiento relacionadas con las ciencias de la salud. La publicación de ensayos clínicos sobre $\mathrm{HaD}$ se mantiene constante durante el tiempo, sin identificarse grandes grupos de referencia. Los descriptores y las áreas temáticas son acordes al área estudiada y los ensayos están publicados en revistas relacionadas con el tema a estudio, aunque sería conveniente tener una mayor presencia en publicaciones referentes sobre $\mathrm{HaD}$.

\section{BIBLIOGRAFÍA}

1. Wanden-Berghe C, Martín-Rodero H. 25 años de investigación en nutrición y alimentación en el espacio iberoamericano del conocimiento. Nutr Hosp. 2012;27(Supl 2):26-33. DOI: 10.3305/ nh.2012.27.sup2.6270; PMID: 23568394

2. Sanz-Valero J, Wanden-Berghe C. Análisis bibliométrico de la producción científica, indizada en MEDLINE, sobre los servicios de salud proporcionados por las unidades de hospitalización a domicilio. Hosp Domic. 2017;1(1):21. DOI: 10.22585/hospdomic.v1i1.3

3. Allen L, Jones C, Dolby K, Lynn D, Walport M. Looking for landmarks: the role of expert review and bibliometric analysis in evaluating scientific publication outputs. PLoS ONE. 2009;4(6):e5910. DOI: 10.1371/journal.pone.0005910; PMID: 19536339 
4. González Sánchez F, Pulsant Terry JG, Tito Goire C, Oliva Prevots M, Cintra Castillo M. Análisis de algunos indicadores bibliométricos de la Revista Información Científica de la Universidad Médica de Guantánamo. Revista Información Científica. 2018;97(6):1088-99.

5. López Ferrer M. La evaluación de la actividad científica en las instituciones y el catálogo de indicadores bibliométricos de la URICI en el CSIC [monografía en Internet]. Madrid, España: CSIC - Unidad de Recursos de Información Científica para la Investigación (URICl); 2015 [citado 31 de agosto de 2019]. Disponible en: http://digital.csic.es/handle/10261/126178

6. Valle Díaz de la Guardia AM, Martín García A, Calleja Hernández MÁ, Cabeza Barrera J. Investigación clínica: el ensayo clínico. En: Martínez Nieto C, editor. Ensayos Clínicos: Actualización en ética, normativa, metodología y nuevas tecnologías. Madrid, España: Sociedad Española de Farmacia Hospitalaria SEFH; 2017. p. 25-48.

7. Gálvez Toro. Enfermería Basada en la Evidencia: Cómo incorporar la investigación a la práctica de los cuidados - Evaluación de la calidad de las evidencias científicas [monografía en Internet]. Granada, España: Fundación Index; 2007 [citado 31 de agosto de 2019]. Disponible en: http:// bit.ly/2IAMW8U

8. Manterola C, Asenjo-Lobos C, Otzen T. Jerarquización de la evidencia: Niveles de evidencia y grados de recomendación de uso actual. Rev Chil Infectol. 2014;31(6):705-18. DOI: 10.4067/ S0716-10182014000600011

9. Sanz-Valero J, Tomás-Gorriz V, Morales-Suárez-Varela M. Estudio bibliométrico de la producción científica publicada por la revista Ars Pharmaceutica en el periodo 2001 a 2013. Ars Pharm. 2014;55(2):1-10

10. Brooten D, Roncoli M, Finkler S, Arnold L, Cohen A, Mennuti M. A randomized trial of early hospital discharge and home follow-up of women having cesarean birth. Obstet Gynecol. 1994;84(5):832-8. PMID: 7936522

11. Bernabeu-Martínez MA, Sanz Valero J. Análisis bibliométrico y temático de la producción científica existente en la base de datos bibliográfica MEDLINE sobre medicamentos peligrosos en las Unidades de Hospitalización a Domicilio. Hosp Domic. 2018;2(3):101-15. DOI: 10.22585/ hospdomic.v2i3.50

12. Dickersin K, Manheimer E, Wieland S, Robinson KA, Lefebvre C, McDonald S. Development of the Cochrane Collaboration's CENTRAL Register of controlled clinical trials. Eval Health Prof. 2002;25(1):38-64. DOI: 10.1177/016327870202500104; PMID: 11868444

13. Organización Mundial de la Salud (OMS). Promover la seguridad de los medicamentos para niños. Edición española. Ginebra, Suiza: OMS; 2007.

14. Camargo Jr KR de, Coeli CM. Multiple authorship: growth or inflationary bubble? Rev Saude Publica. 2012;46(5):894-900. PMID: 23128267

15. Franco-Pérez AM, Sanz-Valero J, Wanden-Berghe C, Melian-Fleitas L. La producción científica iberoamericana en ciencias de la nutrición: La indización en PubMed y Google Scholar. Nutr Hosp. 2014;30(5):1165-72. DOI: 10.3305/nh.2014.30.5.7723; PMID: 25365023

16. Domingo-Pueyo A. Análisis bibliométrico de la producción científica española, indizada en MEDLINE, sobre servicios de atención a domicilio provisto por hospital. Hosp Domic. 2017;1(3):14151. DOI: 10.22585/hospdomic.v1i3.24

17. Franco-López A, Sanz-Valero J, Culebras J. Publicar en castellano, o en cualquier otro idioma que no sea inglés, negativo para el factor de impacto y citaciones. JONNPR. 2016;1(2):65-70. DOI: 10.19230/jonnpr.2016.1.2.1005 
18. Arnett JJ. The neglected 95\%: why American psychology needs to become less American. Am Psychol. 2008;63(7):602-14. DOI: 10.1037/0003-066X.63.7.602; PMID: 18855491

19. Sanz-Valero J, Tomás-Casterá V, Tomás-Gorriz V. Estudio bibliométrico de producción y consumo de la revista Farmacia Hospitalaria (2004-2012). Farm Hosp. 2014;38(1):1-8. PMID: 24483853

20. Brase J, Lautenschlager M, Sens I. The tenth anniversary of assigning DOI names to scientific data and a five year history of DataCite. D-Lib Magazine. 2015;21(1-2). DOI: 10.1045/january2015-brase

21. Sanz-Valero J, Gil Á, Wanden-Berghe C, Martínez de Victoria E; Grupo de Comunicación y Documentación Científica en Nutrición (CDC-Nut SENPE). Análisis bibliométrico y temático de la producción científica sobre ácidos grasos omega-3 indizada en las bases de datos internacionales sobre ciencias de la salud. Nutr Hosp. 2012;27(Supl 2):41-8. DOI: 10.3305/nh.2012.27. sup2.6272; PMID: 23568396

22. Leimu R, Koricheva J. Does Scientific Collaboration Increase the Impact of Ecological Articles? BioScience. 2005;55(5):438. DOI: 10.1641/0006-3568(2005)055[0438:DSCITI]2.0.CO;2

23. Hart RL. Collaboration and Article Quality in the Literature of Academic Librarianship. The Journal of Academic Librarianship. 2007;33(2):190-5. DOI: 10.1016/j.acalib.2006.12.002 